https://doi.org/10.18778/2196-8403.2020.02

ELKE MEHNERT

\title{
Die drei Bäume von ANNA SEGHERS (Variationen über Furcht)
}

Als ANNA SEGHERS 1940 mit ihrer Familie versuchte, aus dem von der deutschen Wehrmacht besetzten Teil Frankreichs zu fliehen, schrieb sie Die drei Bäume, drei kurze Texte, die sich mit Angstgefühlen auseinandersetzen - es sind die Angst vor Verfolgung, vor Führungslosigkeit und vor Entfremdung. In der SEGHERS-Forschung haben diese kurzen Geschichten kaum Beachtung gefunden - sehr zu Unrecht, wie die Verfasserin anhand ihrer jahrzehntelangen Erfahrungen im Umgang mit den kurzen Geschichten darstellt.

Schlüsselwörter: ANNA SEGHERS, Anekdotentriptychon, Exilliteratur, Rezeptionsbedingungen, strukturell-funktionale Textanalyse

\section{Die drei Bäume by ANNA SEghers (Variations on Fear)}

In 1940, when ANNA SEGHERS and her family tried to flee the part of France occupied by the German Wehrmacht, she wrote Die drei Bäume; three short texts dealing with feelings of fear - that is, the fear of persecution, of lack of leadership, and of alienation. In research into SEGHERS, these short stories have received little attention - unjustifiably so, as the author shows, based on her decades of experience in dealing with the short stories.

Keywords: ANNA SEGHERS, anecdotal triptych, exile literature, conditions of reception, structural-functional text analysis

\section{Die drei Bäume ANNY SEGHERS (Odmiany lęku)}

Gdy ANNA SEGHERS razem ze swoją rodziną próbowała w 1940 r. uciec z zajętych przez niemiecki Wehrmacht terenów Francji, napisała trzy krótkie teksty Die drei Bäume (Trzy drzewa). Teksty te zajmują się lękiem i pytają, w jaki sposób można sobie z nim radzić - chodzi o lęk przed prześladowaniem, lęk przed brakiem osoby, której można zaufać i lęk przed wyobcowaniem. Literatura naukowa o SEGHERS nie zwróciła uwagi 
na te teksty - niesłusznie. Autorka artykułu przywraca je pamięci opierając się na kilkudziesięcioletnich badaniach poświęconych krótkim opowiadaniom.

Słowa klucze: ANNA SEGHERS, anekdota, literatura emigracyjna, recepcja, strukturalno-funkcjonalna analiza tekstu

\section{Ein Text - verschiedene Rezeptionsbedingungen}

Es gibt Texte, die den Leser*innen durch sein ganzes Leben begleiten und von ihm wiederholt und jeweils anders rezipiert werden. Mir geht es so mit den drei Kurzgeschichten von ANNA SEGHERS, die sie unter dem Titel Die drei Bäume zusammengefasst hat.

Zuerst stieß ich auf sie eher zufällig im Kontext der Beschäftigung mit Wirkungsbesonderheiten poetischer Texte, wie sie Gotthard Lerchner und HansGeorg Werner seit 1970 theoretisch untersucht und exemplarisch erörtert hatten (vgl. LERCHNER 1984; WERNER 1984). Die kurzen SEGHERS-Texte boten sich für die Erprobung dieses - in der DDR-Germanistik bis dato unüblichen - strukturell-funktionalen Verfahrens an.

Als endlich im Nachgang der 1977er Kleist-Konferenz in Frankfurt a.O. zu Beginn der 1980er Jahre auch in der DDR der Weg zu einem erweiterten Erbverständnis geöffnet wurde, interessierte es mich nachzusehen, wie ANNA SEGHERS Elemente der christlichen und antiken Mythologie rezipiert und in eigenen Texten produktiv gemacht hatte. Wiederum lasen sich Die drei Bäume neu.

Die friedliche Revolution 1989 stürzte viele Denkmale vom Sockel - auch die Nestorin der sozialistischen deutschen Nationalliteratur traf dieses Schicksal. Dazu hat im konkreten Fall eine Lesung aus der Autobiographie des ehemaligen Aufbau-Verlagsdirektors Walter Janka beigetragen, die am 28.10.1989 im Deutschen Theater zu Berlin stattfand. Er warf SEGHERS Feigheit vor: In seinem Prozess 1956 hätte sie geschwiegen, anstatt sich auf seine Seite zu stellen. War die Autorin tatsächlich eine furchtsame Person? Hatte sie nicht auf dem Weg ins mexikanische Exil zahlreichen Gefahren mit unglaublichem Mut widerstanden? Da sich ANNA SEGHERS kaum autobiographisch geäußert hat, war eine Antwort auf diese Frage nur in Texten aus jener Zeit zu finden - beispielsweise in Die drei Bäume.

In den 1990er Jahren verschwanden im vereinigten Deutschland nicht nur sozialistische Denkmale aus dem öffentlichen Raum - es verschwanden in den neuen Bundesländern auch viele Texte von DDR-Autor*innen aus den Schul- 
lehrplänen und aus dem germanistischen Curriculum. Hatten sie denn ihre ästhetische Qualität verloren? Oder standen die Rezeptionsweisen der vergangenen Jahrzehnte zwischen Texten und Leser*innen? Um das zu erfahren, bot ich ein SEGHERS-Hauptseminar an, in dem unter anderen Texten auch Die drei Bäume besprochen wurden. Die Studierenden lasen sie mit großem Interesse als den Versuch, eine existenzielle Gefahr mit den Mitteln der Verfremdung zu bewältigen.

Die jüngsten Erfahrungen mit diesen kurzen Prosatexten machte ich kürzlich in einem Gesprächskreis von Literaturfreunden verschiedenen Alters, unterschiedlicher Berufe und Herkunft. Auch ohne Kenntnis des Entstehungskontextes stellten sie sofort eine Beziehung zur Flüchtlingskrise der Gegenwart her und erkannten, dass verschiedene Varianten von Furcht die thematische Klammer der drei Texte bildeten.

Ganz offensichtlich ist es ANNA SEGHERS gelungen, eine menschheitliche Grundsituation so allgemeingültig zu beschreiben, dass sich zu verschiedenen Zeiten Leser*innen verschiedener Generationen, verschiedener Herkunft und mit unterschiedlichen Lese- und Lebenserfahrungen in den Rezeptionsprozess einbringen und zu Cofabulierern werden können.

Diesen Prozess habe ich über mehr als vier Jahrzehnte verfolgt. In dieser Zeit haben sich nicht die Texte, wohl aber ideologische und methodologische $\mathrm{Zu}$ gänge zu ihnen verändert. Davon will ich berichten. Als Zeitzeugin komme ich nicht umhin, häufig in der ersten Person Singular zu schreiben; denn ich will und kann meine individuellen Rezeptionserfahrungen nicht verallgemeinern.

\section{Ängste einer Emigrantin}

Als ich mich vor mehr als vier Jahrzehnten - in anderer Zeit - zum ersten Mal mit diesen Texten befasste, hatten sie bisher kaum im Focus von Interpreten gestanden. An ihrer Zugänglichkeit kann es nicht gelegen haben, denn nach der späten Erstveröffentlichung ${ }^{1}$ wurden sie wieder abgedruckt im Band 1 des Bienenstocks (1953), 1963 in Band 1 der dreibändigen Ausgabe von Erzählungen bei Aufbau, 1964 im Band 1 der zweibändigen Erzählungsausgabe von Luchterhand, 1965 in Band 475 der Inselbücherei (zusammen mit anderen sagen- und legendenhaften Texten), 1975 in einem der wohlfeilen Taschenbücher des AufbauVerlags Berlin und Weimar. Die SEGHERS-Biographien von Kurt Batt, Tamara

1 Sie erschienen 1946 im letzten Heft der in Mexiko herausgegebenen Exilzeitschrift Neues Deutschland. 
Motyleva und der Band Seghers aus der (für Schüler bestimmten) Reihe Schriftsteller der Gegenwart führen diese Texte zwar mit ihrem Entstehungsdatum (und mehrfach mit unkorrektem Titel) an, gehen aber inhaltlich kaum auf sie ein. Die Ursachen können vielfältig sein: Zum Ersten gehörten weder Bibel noch antike Mythen zum ,Traditionskern der erbenden Arbeiterklasse“ (wie in den 1970er/1980er Jahren der Terminus technicus in der Erbedebatte lautete). Also fanden sie in Schulen und germanistischem Curriculum kaum Erwähnung. Ohne Kenntnis der Odyssee und des Alten Testaments fehlten aber wichtige Zugänge zum Baum des Odysseus und dem Baum des Jesaja. Für möglich, wenngleich weniger bedeutungsvoll halte ich (zweitens) Unsicherheiten bezüglich der Genreeinordnung. ${ }^{2}$ ANNA SEGHERS selbst hatte sich dazu nicht festgelegt - wohl auch, weil für sie Genrefragen (wie sie Anfang der 1960er Jahre gelegentlich einer Diskussion im legendären Leipziger Hörsaal 40 kundtat) irrelevant waren. Am wahrscheinlichsten erscheint mir (drittens), dass Die drei Bäume nicht in das DDR-offiziell verbreitete heroische Bild von SEGHERS, der ,sozialistischen Kämpferin', passten, denn es sind Variationen über Furcht. ${ }^{3}$

Ob die Autorin selbst eine ängstliche Person gewesen sei, war nicht erst im Wendeherbst eine viel diskutierte Frage. Ich erinnere mich an ein Gespräch in der SEGHERS-Gedenkstätte am 20.01.1989, als darüber diskutiert wurde, ob SEGHERS mutig gewesen sei. Die Gesprächsteilnehmer - darunter der Leiter des Archivs und mehrere Literaturwissenschaftler*innen - waren einhellig der Auffassung, sie habe ihre Meinung stets vertreten, jedoch (wenn diese nicht mit der Parteilinie übereinstimmte) nicht öffentlich polemisiert, sondern Kontakt zu den ,entsprechenden Stellen' gesucht. ${ }^{4}$ Zehn Monate später wurde diese

2 Man findet sowohl die Bezeichnung ,Kurzgeschichten“als auch Geschichten, Erzählungen, Anekdoten in der Sekundärliteratur.

3 Ich beziehe dabei meinen im Jahr 1983 in der Wissenschaftlichen Zeitschrift der PH Ernst Schneller 2/1983:37-42 veröffentlichten Aufsatz sowie meine Habilitationsschrift Nationales und Internationales in den Traditionsbeziehungen von Anna Seghers (Potsdam 1980, Typoskript) in die Ausführungen ein.

$4 \quad$ Als Belege wurden zahlreiche Fakten angeführt; beispielsweise habe SEGHERS nach der Biermann-Affäre 1976 den Ausschluss Christa Wolfs aus dem Schriftstellerverband der DDR verhindert und ein Verbot von Strittmatters Roman Ole Bienkopp erfolgreich abgewehrt. 
Frage öffentlich gestellt. Ausgelöst hatte das die Lesung aus Walter Jankas ${ }^{5}$ Autobiographie am 28. Oktober 1989 im Deutschen Theater zu Berlin. Rolf Schneider schreibt dazu:

Das Buch Schwierigkeiten mit der Wahrheit verdankte seine Wirkung in der DDR auch dem Umstand, dass hier ein Monument Schaden nahm. Geschildert wird, wie die Schriftstellerin Anna Seghers, in Sorge um ihren Freund Georg Lukacs, im Oktober 1956 Janka dazu bewegte, den ungarischen Philosophen aus dem von Wirren heimgesuchten Budapest in die DDR zu holen. Becher stimmte zu. Die Reisevorbereitungen liefen an. Ulbricht untersagte das Vorhaben. (SCHNEIDER 1990: o.S.)

Im Prozess, schreibt Janka, sei der Vorgang gegen ihn verwendet und als versuchter konterrevolutionärer Akt dargestellt worden. Die im Zuschauerraum anwesende ANNA SEGHERS habe nicht die mindeste Regung eines Protestes von sich gegeben (vgl. SCHNEIDER 1990). ${ }^{6}$ Noch einmal Janka dazu: „Gerade sie hätte sich der Mitverantwortung nicht entziehen dürfen. Schon deshalb nicht, weil sie die namhafteste Frau war, die es sich leisten konnte, ihre Stimme der Wahrheit zu leihen“"(JANKA 1990:93).

\section{Politische Wende und Kanon}

Schon nach der Reformation im 16. Jahrhundert verschwanden Heiligenstatuen in sogenannten Götzenkammern. Ähnliches geschah jetzt mit den Ikonen der sozialistischen deutschen Nationalliteratur. Die Literaturgeschichte las sich über Nacht anders; DDR-Literatur wurde aus den Regalen der Volksbuchhandlungen und aus öffentlichen Bibliotheken entfernt. Aspekte dieses Vorgangs sind als ,deutsch-deutscher Literaturstreit“ erinnerlich und sollen hier nicht ausführlicher erörtert werden. Aber schon ehe Christa Wolf und Stephan Hermlin unter Rechtfertigungsdruck gerieten, betraf dieses ,Großreinemachen“ ANNA SEGHERS. Schneider berichtet zwar (und hier setzt er die Akzente anders als Janka), dass dabei die gescheiterte Rettungsaktion für Lukacs im Prozess gegen den

5 Walter Janka war bis 1956 Chef des Aufbau-Verlags, dessen Gründung auf eine Initiative Johannes R. Bechers, des Kulturbund-Vorsitzenden, zurückging. Aufbau galt als der renommierteste DDR-Verlag, zu dessen Hausautoren beispielsweise SEGHERS, Becher und Brecht gehörten.

6 Der DDR-Schriftsteller Rolf Schneider über die Prozesse gegen Janka, Harich und andere: Spiegel_1990_23_13499360.pdf (29.01.2020). 
Aufbau-Chef eine marginale Rolle gespielt habe. Aber im Herbst 1989 wurde das Schweigen der SEGHERS zum willkommenen Anlass eines Autodafés. ${ }^{7}$

Als ich Anfang November 1989 in die Volkswohlstraße 81 (SEGHERS' letzte Wohnung und heutige Gedenkstätte) kam, herrschte dort große Aufregung. Die Schränke im Arbeitszimmer der Autorin (damals noch Aufbewahrungsort von Mappen mit Typoskripten und Briefen) wurden nach entlastendem Material durchsucht. Freilich hätte man wissen können, dass SEGHERS nach den Erfahrungen der Gestapo-Besuche in ihrer Pariser Wohnung, dem möglicherweise inszenierten Unfall in Mexiko, den ZK-Verdächtigungen gegen Noel H. Field als amerikanischem Agenten ihre Lektion gelernt hatte. Weder be- noch entlastende Notizen irgendwelcher Art fanden sich - es sei denn, man betrachtete den bis dato unveröffentlichten Text Der gerechte Richter (SEGHERS 2002) als Kommentar zu den Vorgängen von 1956. So blieb öffentlich unwidersprochen, was Janka in seiner Autobiographie über die mangelnde Solidarität der Vorsitzenden des Schriftstellerverbandes geschrieben hatte. ${ }^{8}$

Jankas Verdikt wirkte nachhaltig. Noch im Jahr 2000, dem Jahr des 100. Geburtstags von ANNA SEGHERS, als sich die Studenten meines Hauptseminars in der Berliner Gedenkstätte mit Ruth Radvanyi ${ }^{9}$ trafen, waren sie darüber verblüfft, dass diese erklärte, sie müsse jetzt vieles nachholen, um ein genaueres Bild der Mutter zu bekommen: Sie läse zum ersten Mal deren Texte und besuche Zeitzeugen, weil sie Authentisches über die Rolle der Mutter im Janka-Prozess erfahren wolle. Das von Janka gezeichnete Porträt einer feigen, nur auf das unbeschädigte eigene Image bedachten Frau passe so gar nicht zu ihren Erinnerungen:

Für meinen Bruder Pierre und mich war Anna Seghers nicht die berühmte Schriftstellerin, sondern unsere Mutter. Wir wußten, daß sie schrieb, daß Schreiben ihre Arbeit war, und trotzdem gehörte sie ganz uns, in einer Zeit, in der wir uns täglich, stündlich gemeinsam Sorgen um unseren eigenen Alltag und um die Welt machten. (RADVANYI 2000:105)

7 Einen interessanten Zeitzeugenbericht verdanken wir Christel Berger, der ersten Co-Vorsitzenden der ANNA SEGHERS-Gesellschaft, die sich aus dem Abstand von zwei Jahrzehnten an die Aufregung um ANNA SEGHERS' materielles und ideelles Erbe im Wendeherbst 1989 erinnert (BERGER 2011).

8 Allerdings bekam ich damals Postkartengrüße von Janka an SEGHERs in die Hand, die eine andere Sprache sprachen. Janka war nach seiner Haft Dramaturg bei der DEFA und an SEGHERS-Verfilmungen beteiligt.

9 Ruth Radvanyi, die Tochter von ANNA SEGHERS, war Chefärztin einer Berliner Kinderklinik und hatte sich nach eigener Aussage bisher so gut wie gar nicht für die Bücher ihrer Mutter interessiert. 
Im Jahr 2000 bestand also immer noch Aufklärungsbedarf hinsichtlich der Rolle von ANNA SEGHERS in den ideologischen Auseinandersetzungen des 20. Jahrhunderts. Beispielsweise hatte im Novemberheft des Journals Literaturen Frauke Meyer-Gosau unter der Überschrift Die Frau mit der Maske. Übermutter und Ikone: Anna Seghers zum 100. Geburtstag ein ,Unanständchen“ angestimmt, in dem sie die Jubilarin als „beinharte Sozialistin“ apostrophierte, die „als Mitglied der kommunistischen Partei seit 1928 [...] auch teil [hatte] an der innerstaatlichen und -parteilichen Vernichtungspolitik, die der Stalinismus [...] exekutierte - nicht nur eine Zeit-Zeugin also, auch eine politisch involvierte“ (MEYERGOSAU 2000:93). ${ }^{10}$

Auch ich hatte den 100. Geburtstag der Autorin zum Anlass erneuter Beschäftigung mit SEGHERS-Texten genommen und ein Hauptseminar angeboten, weil ich wissen wollte, ob man ein reichliches Jahrzehnt nach der politischen Wende (und Jankas Anklage) die ideologische Blockade durchbrechen und den Blick wieder auf die ästhetische Qualität von SEGHERS' Texten richten könnte. ${ }^{11}$ Damit befand ich mich ganz auf der Linie von Reich-Ranicki, der sich schon 1991 an die Seite von SEGHERS gestellt hatte:

Text bleibt Text - davon waren wir seit eh und je überzeugt. Gilt der Satz nicht mehr? Doch, er gilt nach wie vor. Aber wahr ist auch, daß literarische Arbeiten im Laufe von Jahren und Jahrzehnten verblassen und absterben, daß sie andererseits in einer neuen Situation eine neue Bedeutung gewinnen können, ja sogar eine ungeahnte Aktualität. (REICH-RANICKI 1991:46)

Bestärkt sah ich mich in meinem Vorhaben auch durch eine Bemerkung von ANNA SEGHERS gegenüber Pierre Durant: „Wissen Sie, wenn man ein Buch in der Schule studiert, riskiert man es sehr, den Geschmack daran zu verlieren. Man seziert darin alle Sätze, man erforscht darin alle Nuancen, und das wird

10 Es ist mir kein weiteres Beispiel dafür erinnerlich, dass sich meine Studenten über einen Zeitschriftenaufsatz so empört hätten, dass sie an die Redaktion geschrieben hätten: „Unserer Meinung nach haben Sie viel zu wenig zum Gesamtwerk und zum Leben der Autorin gesagt. Der einzig erkennbare Schwerpunkt ist Anna Seghers' politische Gesinnung“ (Aus dem Brief des SEGHERS-Hauptseminars 2000 an die Redaktion von Literatuen). Dieser Brief wurde zwar teilweise abgedruckt - die Einladung der Studenten zur Diskussion an der Chemnitzer Universität jedoch dankend abgelehnt.

11 Nichts anderes hatte Marcel Reich-Ranicki in dem 1990 geschriebenen Aufsatz Nicht gedacht soll ihrer werden? beabsichtigt, als er schrieb: „Haben wir Anna Seghers geschätzt und bewundert, weil wir sie für edel, hilfreich und gut hielten? Oder weil wir ihr die Romane Transit und Das siebte Kreuz verdanken [...]? Haben sie sich denn in der Zwischenzeit verändert?“" (REICH-RANICKI 1991:46). 
schrecklich langweilig“ (DURANT 1976:5). Ich hatte diese Erfahrung während der eigenen Schulzeit im Deutschunterricht gemacht und orientierte daher meine Textauswahl nicht auf die kanonischen, durch die Rezeptionsweisen der vergangenen Epoche belasteten Werke, sondern wählte wenig besprochene, in Sujet- und Traditionswahl oftmals überraschende Texte aus, die sich schwerlich in das vom Unterricht an DDR-Schulen vermittelte Bild der Autorin einfügten.

\section{Kritik früherer Rezeptionsweisen - Erfahrungsbericht}

Ich wollte die Schriftstellerin als eine sozial denkende Frau aus bürgerlichem Haus zeigen, die den Mut hatte, ihren eigenen Weg zu gehen; die eine liebevolle Mutter war, eine tolerante Ehefrau, die ein Faible für nahrhaft besetzte Kaffeetische hatte und zugleich ein öffentliches Leben führte, in dem die Tage , dicht besetzt' waren. Es war doch nicht falsch, was der Deutsch-Lehrplan für Klasse 6 zu der Erzählung Das Duell ausführte (LEHRPLAN 1987:163), ${ }^{12}$ es stimmt ja, dass sich SEGHERS ,früh der revolutionären Arbeiterbewegung anschloss, am antifaschistischen Kampf im Exil teilnahm und in der Weltfriedensbewegung aktiv mitarbeitete" (LEHRPLAN 1987:91). Aber das war nur eine, die öffentliche Seite der Autorin. Dass es auch eine private Seite gab, blieb in der DDR sowohl der Öffentlichkeit als auch den Schülern verborgen; dabei hat SEGHERS auch persönliche Sorgen gehabt und war oft bekümmert - über den Tod der Eltern, die fragile Sicherheit ihrer Familie, fehlendes Geld, verlorenes Gut und vage Arbeits- wie Heimkehrmöglichkeiten nach dem erhofften Ende der Nazidiktatur. Es sind das die ganz gewöhnlichen alltäglichen Ängste einer Frau und Mutter, verstärkt durch die Tatsache, dass sie Jüdin, KPD-Mitglied und Ehefrau eines ungarischen Spanienkämpfers war. Davon zeugen Die drei Bäume aus dem Jahr 1940 - die Variationen über Furcht.

Man sollte annehmen, dass in den letzten drei Jahrzehnten gerade deswegen Die drei Bäume Aufmerksamkeit gefunden hätten - stützen sie doch das 1989 von Janka geprägte, so nachhaltig wirksame Bild von der angstvollen, ja feigen, selbst mit ihren Genossen wenig solidarischen Staatsdichterin. Aber das ist bis heute nicht geschehen. Nach wie vor spielen diese Texte in der SEGHERS-

12 In der Erzählung komme „,das Vertrauen in die Kraft der Menschen zum Ausdruck, aus der Vergangenheit des Faschismus zu lernen und ein neues Leben aufzubauen“ (LEHRPLAN 1987:163). 
Rezeption eine marginale Rolle. ${ }^{13}$ Dabei ist in den letzten Jahren vieles aus dem persönlichen Leben der Autorin (gerade während der Exilzeit und in den ersten Jahren nach dem Krieg) bekannt geworden, das einen genaueren Kontext zu den Drei Bäumen konturiert und damit zu ihrer historisch-genetischen Einordnung beiträgt. Es lohnt also, mit diesem Wissen versehen diese Prosatexte wieder - und vielleicht anders zu lesen als zur Zeit ihrer ersten Veröffentlichung. Pierre Radvanyis Erinnerungen an seine Mutter (RADVANYI 2006) sind dafür besonders hilfreich. Er schildert den Sommer 1940, als Mutter und Kinder aus Paris fliehen, dann noch einmal zurückkehren, ohne sich jedoch in die inzwischen observierte Wohnung zu trauen:

Am nächsten Morgen beschlossen wir, so bald als möglich nach Freunden zu suchen, die Paris vielleicht nicht verlassen hatten oder wie wir zurückgekehrt waren. Wir hatten kaum noch Geld und mußten unbedingt einen Weg aus der Falle finden, in die wir nun geraten waren. (RADVANYI 2006:48)

Erst in letzter Minute und mit dem Erlös verpfändeten Schmucks gelingt die Flucht in die unbesetzte Zone - aber auch sie ist keine sichere Zuflucht. Darauf weist Christiane Zehl-Romero hin:

Die Bedrohung war eine reale: Der Artikel 19, Abs. 2 des Waffenstillstandsvertrages hob das Asylrecht in ganz Frankreich auf. Alle Personen deutscher Abstammung waren auszuliefern, wenn die deutsche Regierung sie namentlich anforderte. (ZEHL-ROMERO 2000:359)

So also müssen wir uns den historischen Entstehungskontext der Drei Bäume vorstellen. Gut möglich, dass ANNA SEGHERS versucht hat, ihrer Angst schreibend ein Ventil zu schaffen. ${ }^{14}$ Dabei mag eine Rolle gespielt haben, dass sie sich schon früh mit Angstgefühlen auseinandergesetzt hat. Zehl-Romero hat bemerkt, dass in der SEGHERSSCHEN Privatbibliothek auffällig viele Schriften von Kirkegaard stehen, deren Widmungen auf intensive Lektüre in den 1920er Jahren hindeuten:

Bei Kirkegaard ist die Grunderfahrung des Menschen die Angst, der er die Begriffe der Freiheit und radikalen Entscheidung zuordnet. Erlösung aus der Verzweiflung, der Unsicherheit und der Einsamkeit menschlicher Existenz, aus der ,Entfremdung des Menschen, ist allein durch die Gnade Gottes möglich. Es war eine Botschaft, deren Unbedingtheit zu Netty Reiling sprach, wobei sie zumindest bis 1925 noch

13 Selbst im Argonautenschiff von 2007, das eine Jahrestagung der SEGHERS-Gesellschaft zum Thema ,Märchen, Sagen, Mythen und Legenden im Werk von ANNA SEGHERS“ dokumentiert, werden Die drei Bäume nur erwähnt, aber nicht besprochen.

14 In dem Roman Transit heißt es: „Denn abgeschlossen ist, was erzählt ist“ (SEGHERS 1951:201). 
mit dem Glauben oder der Sehnsucht nach dem Glauben an einen Gott lebte. (ZEHL-ROMERO 2000:105)

SEGHERS ist von Reich-Ranicki einmal als „Fideistin“ apostrophiert worden (REICH-RANICKI 1963:357) - eine sicherlich nicht zutreffende Wertung. Aber schließt ihre Entfremdung vom jüdischen Glauben denn Sehnsucht nach göttlicher Führung, ja, überhaupt einer Wegweisung in unübersichtlichen Zeiten aus? Höchstwahrscheinlich ist der jungen NETTY REILING die Märtyrerlegende um den Propheten Jesaja bekannt gewesen. Nicht nur in religionsgeschichtlichen Schriften, sondern auch im Talmud und einer Bibel von SEGHERS stecken Merkzettel - Spuren wiederholter Lektüre.

\section{Ausgeschlagenes Erbe?}

Weder Bibel noch Talmud oder antike Autoren gehörten bis in die 1970er Jahre zum anzueignenden Erbe in der realsozialistischen Gesellschaft. „Die Entwicklung eines wissenschaftlichen Weltbildes hat die Mythologie als voroder unwissenschaftliche Erklärung des Lebensprozesses verdrängt und überflüssig gemacht“" (DAHNKE 1977:74), schrieb Hans-Dietrich Dahnke rückblickend. Nun aber wurde das Erbproblem zum Feld ideologischer Auseinandersetzung erklärt und der Traditionskanon erweitert. Sowohl die Mythen der Bibel als auch antike Philosophie und Literatur fanden Eingang in das germanistische Curriculum. Damit entfielen in den 1980er Jahren bestimmte Rezeptionshindernisse, und ich erprobte den erweiterten Traditionshorizont, indem ich Die drei Bäume auf den Lektüreplan eines Seminars zur Gegenwartsliteratur setzte. Die Bibel als Sekundärquelle für ein Literaturseminar war in den 1980er Jahren durchaus unüblich. Aber mit Hilfe einer Konkordanz ließen sich meine - mehrheitlich kirchenfernen - Studenten auf das Neuland ein.

Der biblischen Überlieferung nach hat Jesaja nicht nur im achten Jahrhundert vor unserer Zeit die Geburt Christi vorausgesagt, sondern er hat auch Menasse, den König von Judäa, kritisiert und Jerusalem unter dessen Herrschaft mit Sodom und Gomorrha verglichen. König Menasse entledigt sich seines Kritikers, indem er den flüchtigen, führungslos gewordenen Propheten samt seinem Versteck - einem hohlen Baumstamm - zersägt (vgl. DUCHET-SUCHAUX / PASTOUREAUX 2005:163). SEGHERS geht mit der biblischen Überlieferung frei um - übernimmt lediglich die Beschreibung auswegloser Angst - adäquat der eigenen Situation im Sommer 1940. Ob man sich (wie Jesaja) unter der, erhabenen Stimme ' eine göttliche vorstellen möchte oder (profaner) die Handlungsanweisung einer Parteiführung - das ist irrelevant. Als diese Stimme nach der Niederlage seines 
Volkes verstummt, verlässt Jesaja, den Propheten, aller Mut, denn er war es gewohnt, „Weisungen zu empfangen. Da fing er an sich zu fürchten“(SEGHERS 1975:60): Jesaja fühlt sich gelähmt in seiner Einsamkeit, er wird handlungsunfähig. SEGHERS beschreibt Entfremdung, ja Verzweiflung eines auf sich selbst zurückgeworfenen Individuums. Könnte das nicht Ausdruck einer Konfession, eines Traumas sein, von dem sich die Erzählerin schreibend zu befreien sucht? Was für den Propheten die Stimme Gottes ist - für die von Mann, Freunden, Genossen getrennte Frau ist es vielleicht (und viel profaner) die fehlende Kommunikation im Moment der Gefahr. Die Gefährdung Jesajas ist nicht eingebildet, sondern real - so real wie für die vaterlose kleine Familie im von deutschen Soldaten besetzten Frankreich. Nach biblischer Überlieferung hat König Menasse sich an seinem Kritiker Jesaja gerächt, indem er selbst ihn samt Zedernstamm zersägt hat. Er hat gewusst, was er tat. Hier weicht SEGHERS von der biblischen Quelle ab. Die Holzarbeiter in ihrer Geschichte ahnen nicht, dass sich im hölzernen Versteck ein Mensch befindet, den sie zersägen. Ihnen kann man keine Schuld geben. Die liegt allein bei dem verstummten, geflüchteten Propheten. Hätte Jesaja doch den Mut aufgebracht sein Versteck zu verlassen! „Er aber fürchtete sich vor den Holzarbeitern“ (SEGHERS 1975:60). Dieses „,er [...] fürchtete sich nicht" wird viermal wiederholt und gewinnt dadurch große Eindringlichkeit. Man fragt sich, warum er die Menschen wohl mehr fürchtet als den (sicheren) Tod? Dass es auf diese Frage nicht nur eine Antwort gibt und dass diese zu unterschiedlichen Zeiten auch unterschiedlich ausfällt, macht den Reiz dieser Bibeladaption aus und führte im Seminar zu lebhaften Diskussionen.

\section{Struktur und Funktion: Der Baum des Ritters}

Der Baum des Ritters ist der erste und zugleich kürzeste von den drei Texten. An diesem Beispiel habe ich mit meinen Studenten den strukturell-funktionalen Interpretationsansatz von LERCHNER (1984) und WERNER (1984) ausprobiert.

Der Text umfasst fünf Sätze: zwei Satzgefüge, eine Satzverbindung, einen einfachen und einen zusammengesetzten Satz. Der erste Satz enthält bereits alle wesentlichen Informationen. Der berichtete Sachverhalt hat sich erst kürzlich an einem genau bestimmten Ort (in den Ardennen) zugetragen: Beim Fällen einer hohlen Buche finden die Waldarbeiter in der Höhlung „einen Ritter in voller Rüstung, kenntlich an seinem Wappen als ein Gefolgsmann Karls des Kühnen von Burgund“" (SEGHERS 1975:59). Damit ist auch gesagt, dass der Ritter vor mehr als viereinhalb Jahrhunderten den Tod gefunden hat. Ein Erzählen auf die Pointe hin (wie es Anekdote oder auch Kurzgeschichte erfordern) ist 
schon nach dem ersten Satz nicht mehr möglich - ein Spannungsbogen lässt sich nicht aufbauen. Immerhin wird die Lesererwartung aufrechterhalten, denn der Rezipient will wissen, wieso der Ritter in den hohlen Baum geraten ist. Der zweite Satz enthält die Antwort: Dieser Ritter hatte sich auf der Flucht vor den Soldaten König Ludwigs des XI. in seiner Todesangst in einen Baum gezwängt. Das wird von einem allwissenden Erzähler mitgeteilt, der seine Informiertheit auch im Folgenden, dem dritten Satz beweist: „Nach dem Abzug seiner Verfolger hatte er sich nicht mehr herausgefunden und war elend zugrunde gegangen in seiner Zuflucht" (SEGHERS 1975:59). Der Berichterstatter weiß nicht nur über Gegenwärtiges Bescheid (kürzlich wurde der hohle Baum gefällt), sondern er berichtet über den Tod eines Ritters vor Jahrhunderten, als sei er Augenzeuge gewesen. Der vierte Satz verbindet die Zeitebenen miteinander: Der Baum „rauschte und grünte weiter, während der Ritter in ihm keuchte, weinte, betete und starb" (SEGHERS 1975:59). Im (scheinbar entbehrlichen) Autorenkommentar des letzten Satzes spekuliert der Erzähler - von der Gegenwart ausgehend - der Baum wäre nämlich weiter gewachsen, hätten ihn die Holzfäller nicht geschlagen. Naturwissenschaftlich gebildete Leser*innen darf daran zweifeln - wird doch einer Buche die Lebenszeit von drei Jahrhunderten zugeschrieben. Aber ist das ein wichtiger Teil der Botschaft? (Wohl kaum.) Da alle wichtigen Informationen scheinbar schon im ersten Satz enthalten sind, muss das in den Sätzen zwei bis fünf Ausgesagte eine nicht auf den ersten Blick sichtbare Bedeutung haben. Wenn man sich - den Anregungen LERCHNERS (1984) und WERNERS (1984) folgend - mit nicht verbalisierten Wertungen im Text beschäftigt und dazu die Topikreihen ,Ritter' und ,Baum' verfolgt, zeigt sich folgende Struktur: Vom Ritter wird ausgesagt, dass er ,,in voller Rüstung“ (Satz 1) war, „,kenntlich an seinem Wappen“ (Satz 1) als „Gefolgsmann Karls des Kühnen von Burgund" (Satz 1). Die Topikreihe wird fortgesetzt durch „Flucht“ (Satz 2), „Todesangst“" (Satz 2), ,in einen Baum gezwängt“ (Satz 2), „nicht mehr herausgefunden“ (Satz 3), ,elend zugrundegegangen“ (Satz 3), „der Ritter [...] keuchte, weinte, betete, starb“ (Satz 4), „der Tote“ (Satz 5). Zur Topikreihe ,Baum' gehören: ,uralter Baumschlag“ (Satz 1), „hohle Buche“ (Satz 2), (in) ,ein(en) Baum“ (Satz 2), „Zuflucht“ (Satz 3), „der Baum, alt und mächtig“ (Satz 4), ,rauschte und grünte weiter“ (Satz 4), ,stark und makellos“ (Satz 5), „Höhlung“ (Satz 5), „wuchs weiter“ (Satz 5), ,setzte Ringe an“ (Satz 5), „,breitete seine Äste“(Satz 5), „,beherbergte Generationen von Vogelschwärmen“ (Satz 5), „wäre weiter gewachsen“ (Satz 5).

Es fällt auf, dass die Topikreihe ,Baum`stärker besetzt ist als die Reihe ,Ritter'. Dominieren in Satz eins noch Informationen über den Ritter, so stehen im 
letzten Satz sieben Informationen über den Baum, aber nur eine, die sich auf den Ritter bezieht. Er wird als „der Tote“ bezeichnet; im Kontrast dazu drücken sechs sprachliche Einheiten die Überlebenskraft des Baumes aus. Im Titel sind beide Topikketten quasi ,pur' genitivisch miteinander verbunden. Es wird der Eindruck des Besitzes erweckt: Der Baum gehört dem Ritter. Die Sätze drei bis fünf kehren allerdings das Possessivverhältnis um: Der Baum überlebt, aber der Gefangene des Baums stirbt. Die Flucht hat nur scheinbar in eine Zuflucht geführt; Sein und Schein widersprechen einander in mehrfacher Hinsicht, denn auch der Baum ist nur scheinbar makellos. Er stand zufällig als ,Zuflucht‘ am Weg des Ritters, wurde zufällig zu dessen Grab. Während die Existenz des Ritters endlich ist, hätte der ,uralte Baum“-auch mit dem in ihm verborgenen Flüchtling - noch weitergelebt, wären die Holzfäller nicht gekommen. So wird die Überlebenskraft der Natur ins Unendliche transformiert und der Endlichkeit des menschlichen Lebens gegenüber gestellt. Der Mensch braucht die Natur - nicht umgekehrt. Vielleicht kann man diesen Text als Elegie lesen - ich zögere ihn tragisch zu nennen; denn von einem Gefolgsmann Karls des Kühnen ist anderes zu erhoffen als die Flucht vor dem Feind. Aber worauf kann man schon setzen in Zeiten wir jenen, die wir als Entstehungszeit des Textes beschrieben haben?

\section{Heimkehr in ein fremdes Land}

Die dritte und längste Geschichte fragt nach dem Danach: Wie wird das Heimkommen sein nach langen Jahren der Flucht? In Odysseus erblickt SEGHERS einen Gefährten, den die Geschichte von ihr getrennt hat: Nach zwei Jahrzehnten erlauben ihm die Götter die Heimkehr. Von seinem Hab und Gut, das die Freier verprasst haben, soll die Rede nicht sein. Aber wie wird ihn seine Frau aufnehmen? Wird sie ihn wiedererkennen nach zwanzig Jahren, die ihn (aber auch sie) verändert haben? Und (wichtiger noch): Wird sie sich zu ihm bekennen? In diesem Text bleibt SEGHERS dicht an der Quelle ihrer Stoffwahl: Penelope stellt Odysseus auf die Probe: Nur er kann wissen, dass er eigenhändig das Ehebett in den Stamm einer mächtigen Buche geschnitten hat. Wenn sie also listig vorschlägt, dem müden Heimkehrer sein Bett ans Feuer tragen zu lassen und er diesen Vorschlag als unrealisierbar zurückweist, so wird klar: Der Heimgekehrte ist tatsächlich der Mann, den Penelope vor zwei Jahrzehnten geheiratet hat. Dieses Wiedersehen vollzieht sich merkwürdig emotionslos - als hätte die Autorin schon 1940 geahnt, dass ihr eigener Mann zwar aus dem Exil zu ihr in die DDR kommt - aber erst 1952 und mit einer Frau an seiner Seite. Darüber 
ist kaum etwas öffentlich geworden; bis zum Tod von Laszlo Radvanyi im Jahr 1978 jedoch lebte das Ehepaar gemeinsam in einer zwar geräumigen, aber bescheidenen Wohnung in der Berlin-Adlershofer Volkswohlstraße 81. Weggefährten bezeugen, dass Laszlo Radvanyi bis zuletzt der erste Leser und Kritiker von Texten seiner Frau geblieben ist.

Ob Odysseus in Ithaka nach seiner Heimkehr zur Ruhe gekommen ist, hat uns Homer verschwiegen. Von SEGHERS' Befindlichkeit in den ersten Nachkriegsjahren wissen wir nur Bruchstückhaftes. In Biographien (vgl. HILZINGER 2000 / ZEHL-ROMERO 2000) wird von Ruhelosigkeit berichtet, sie selbst nennt ihre Tage , dicht besetzt', und aus dem teilweise erschlossenen Briefwechsel wissen wir, dass sie sich im Volk der ,kalten Herzen“ wie in einer neuen Eiszeit gefühlt hat. Der bald einsetzende Streit zwischen den Vertretern der äußeren und der inneren Emigration, das Misstrauen gegenüber West-Emigranten, der unterschwellig vorhandene Antisemitismus - alles das mögen Gründe dafür gewesen sein, dass es SEGHERS nicht leicht gefallen ist, in Deutschland wieder Fuß zu fassen. Zahlreiche öffentliche Verpflichtungen minimieren die Schreibzeit, und der äußere (wohl nicht immer mit dem inneren identische) Auftrag, mit ihren Texten auf die Notwendigkeiten des Tages zu reagieren, lassen sich eher erahnen als mit authentischen Äußerungen der Autorin belegen. Die Literaturkritik aus der (alten) Bundesrepublik Deutschland ist mit den SEGHERS-Werken der Nachkriegszeit (teils berechtigt, teils nicht) wenig sensibel umgegangen. Aber auch die Germanistik in der DDR hat Desiderate hinterlassen - und das betrifft nicht nur solche Texte wie Die drei Bäume. Generell entsteht der Eindruck, dass sich DDR-Literaturwissenschaftler*innen mit Texten schwergetan haben, in denen negative Gefühle oder tragische Konfliktlösungen dargestellt sind. Die Sozialisten liebten das Tragische nicht, weil es ihrem Menschenbild widersprach. Im Standardwerk über marxistische Ästhetik kann man lesen, dass

im Verlauf des sozialistischen Aufbaus die Klassengegensätze verschwinden [...], die tragischen Situationen sozialen Ursprungs zum Gegenstand des historischen Genres werden und sich die tragischen Konflikte aus der Sphäre zwischenmenschlicher Beziehungen in die Sphäre der Beziehungen zwischen Menschen und Natur verlagern. (KAGAN 1969:109)

Scheinbar triumphiert die Natur auch über Jesaja und den Ritter Karls des Fünften von Burgund - aber die Gründe ihres Scheiterns liegen in den Personen selbst, in ihrer Unfähigkeit, Furcht zu überwinden. SEGHERS hat sich in Die drei Bäume wohl die eigene Angst vor Einsamkeit, Verfolgung und Entfremdung von der Seele geschrieben - eine ihr gemäße Form der Katharsis. Entstanden sind Texte, die wiederholter Aneignung standhalten und die den Leser*innen 
immer aufs Neue herausfordern, sich zu ihnen in Beziehung zu setzen. Freilich muss man sie „wach“, ,abtastend“ lesen - nur dann kommt heraus, was die Autorin meint (SEGHERS 1972:120). Diese Erfahrung habe ich in zwei verschiedenen politischen Systemen mit unterschiedlichen Rezipientengruppen gemacht. Davon wollte ich berichten und zugleich ermuntern, den Text beim Wort zu nehmen - nicht die ideologische Bewertung des Verfassers oder der Verfasserin.

\section{Literatur}

Berger, CHRISTEL (2011): Als wir Anna Seghers mit der Gründung einer Gesellschaft ,retten 'wollten, Gemischte Erinnerungen. In: Argonautenschiff 20/2011:277-283.

DAHNKE, HANS-DiETRICH (1977): Erbe und Tradition in der Literatur. Leipzig.

Duchet-Suchaux, Gaston / Pastoureaux, Michel (2005): Lexikon der Bibel und Heiligen. Paris.

DurANT, PIERRE (1976): Der Satz von Pythagoras reicht nicht aus. Gespräch mit Anna Seghers. In: Grazer Tageszeitung v. 30.04.1976:5.

Hilzinger, SONJa (2000): Anna Seghers. Stuttgart.

JANKA, WALTER (1990): Schwierigkeiten mit der Wahrheit. Berlin / Weimar.

KAGAN, MoISSEJ (1969): Vorlesungen zur marxistisch-leninistischen Ästhetik. Berlin.

LEHRPLAN DER ZEHNKLASSIGEN ALLGEMEINBILDENDEN OBERSCHULE, DEUTSCHE SPRACHE UND LITERATUR (1987): Teil Literaturunterricht, Klassen 5-10. Berlin.

LERCHNER, GOTTHARD (1984): Sprachform von Dichtung. Berlin / Weimar.

MEHNERT, ElKe (1980): Nationales und Internationales in den Traditionsbeziehungen von Anna Seghers. Potsdam (Dissertation B, Typoskript).

MeHNERT, ElKe (1983): Variationen über Furcht. In: WZ der PH „Ernst Schneller “ Zwickau 19/H.2:37-42.

Meyer-Gosau, Frauke (2000): Die Frau mit der Maske. In: Literaturen 11:92-94.

RAdVANYI, PIERre (2006): Jenseits des Stroms. Erinnerungen an meine Mutter Anna Seghers. Berlin.

REICH-RANiCKI, MARCEL (1963): Deutsche Literatur in Ost und West. München.

REICH-RANICKI, MARCEL (1991): Nicht gedacht soll ihrer werden? In: Ders.: Ohne Rabatt. Stuttgart, 43-46.

SCHNEIDER, Rolf (1990): Schwierigkeiten mit der Wahrheit: Spiegel_1990_23_ 13499360.pdf (26.01.2020).

SEGHERS, ANNA (1951): Transit. Berlin.

SEGHERS, ANNA (1972): Sonderbare Begegnungen. Berlin / Weimar.

SEgHers, ANNA (1975): Die schönsten Sagen vom Räuber Woynok. Berlin / Weimar. 
SEghers, AnNa (1976): Gespräch mit Pierre Durant. In: Grazer Tageszeitung, 30.04.1976:5. SEGHERS, ANNA (2002): Der gerechte Richter. Berlin / Weimar.

Walter, Hans-Albert (1986): Eine deutsche Chronik. Das Romanwerk von Anna Seghers aus den Jahren des Exils. In: KEIM, ANTON MARIA (ed.): Exil und Rückkehr, Emigration und Heimkehr. Mainz, 85-119.

WERNER, HANS-GEORG (1980): Subjektive Aneignung - objektive Wertung. In: Weimarer Beiträge 26/H.16:140-149.

Werner, Hans-Georg (1984): Text und Dichtung, Analyse und Interpretation. Berlin / Weimar.

Zehl-Romero, Christiane (1993): Anna Seghers mit Selbstzeugnissen und Bilddokumenten. Hamburg.

Zehl-Romero, Christiane (2000): Anna Seghers. Eine Biographie 1900-1947. Berlin. 